\title{
In Peritoneal Dialysis, Is There Sufficient Evidence to Make "PD First" Therapy?
}

\author{
Pranav Dalal, ${ }^{1}$ Harbaksh Sangha, ${ }^{1}$ and Kunal Chaudhary ${ }^{2,1}$ \\ ${ }^{1}$ Division of Nephrology, Department of Internal Medicine, University of Missouri, Columbia, Mo 65212, USA \\ ${ }^{2}$ Division of Nephrology, Harry S Truman Veterans's Hospital, Columbia, Mo 65201, USA \\ Correspondence should be addressed to Kunal Chaudhary, chaudharyk@health.missouri.edu
}

Received 19 January 2011; Revised 6 April 2011; Accepted 20 April 2011

Academic Editor: Hulya Taskapan

Copyright (c) 2011 Pranav Dalal et al. This is an open access article distributed under the Creative Commons Attribution License, which permits unrestricted use, distribution, and reproduction in any medium, provided the original work is properly cited.

\begin{abstract}
Since its introduction more than 3 decades ago, the use of peritoneal dialysis (PD) has increased greatly due to its simplicity, convenience, and low cost. Advances in technique, antibiotic prophylaxis, and the introduction of newer solutions have improved survival, quality of life, and reduced rate of complications with PD. In Hong Kong, approximately 80\% end-stage renal disease (ESRD) patients perform PD; in others, that is, Canada, Australia, and New Zealand, $20 \%-30 \%$ patients use PD. However, in the United States, the annual rate of prevalent patients receiving PD has reduced to $8 \%$ from its peak of $15 \%$ in mid-1980s. PD as the initial modality is being offered to far less patients than hemodialysis (HD), resulting in the current annual incidence rate of less than $10 \%$ in USA. There are many reasons preventing the PD first initiative including the increased numbers of in-center hemodialysis units, physician comfort with the modality, perceived superiority of HD, risk of peritonitis, achieving adequate clearances, and reimbursement incentives to providers. Patient fatigue, membrane failure, and catheter problems are other reasons which discourage PD utilization. In this paper, we discuss the available evidence and provide rationale to support PD as the initial renal replacement modality for ESRD patients.
\end{abstract}

\section{Introduction}

Over half million Americans were undergoing renal replacement therapy (RRT) for ESRD at the end of year 2008. In-center Hemodialysis (HD) and home PD are the two common forms for dialysis therapy. Only about $8 \%$ of patients with ESRD are receiving PD as RRT in the United States compared to Canada and Europe, where PD is much more common [1]. The percentage PD patients has declined significantly from the peak of $15 \%$ in the mideighties to $5.8 \%$ in 2007 [1]. There are numerous factors contributing to the low incidence and prevalence of $\mathrm{PD}$ in this country $[2,3]$. Concerns regarding achievement of adequate clearance with $\mathrm{PD}$, especially in patient with no residual renal function and a perception of better patient survival on HD compared to PD amongst US nephrologists impact selection of dialysis modality. Risk of infectious complications, specially PD peritonitis and catheter-related problems contribute to the selection bias. Systemic factors such as the easy accessibility to HD, financial incentives, and ownership of dialysis units, including units owned by large dialysis organization (LDOs), as well as physician education and exposure to PD during training also play an important role in the selection of RRT for ESRD patients. Strategies to prevent peritonitis, ultrafiltration failure, managing catheterrelated complications, and improving adequacy of dialysis as well as education of patients and medical staff may all help with increasing PD utilization.

\section{Why PD before HD?}

Along with improved survival, other long-term goals of ESRD patients are to improve quality of life, preserve residual renal function, and reduce morbidity. Many young ESRD patients will likely receive $\mathrm{PD}, \mathrm{HD}$, and renal transplantation at different points over their lifetime. Better survival on PD compared to HD during first two years of dialysis treatment is one of the compelling reasons to start patient on PD first. In the last decade, infection-related complications are higher and appear to be increasing in HD patients, whereas such complications are steadily declining in PD patients [1]. Preservation of residual renal function and vascular access 
sites, lower cost of therapy, convenience of home therapy, a flexible schedule, and increased freedom from the patient's perspective are other reasons to offer PD as the first choice modality.

\section{Survival Advantage}

Comparisons of mortality outcomes in HD and PD patients in studies have yielded conflicting results. Vonesh et al. analyzed the Medicare data of 398,340 patients initiating dialysis between 1995 and 2000. They showed survival differences between HD and PD varied substantially according to the underlying cause of ESRD, age, and level of baseline co morbidity. The adjusted mortality rates were either the same or higher for $\mathrm{HD}$ as compared to $\mathrm{PD}$ in most patient groups except in older diabetic patients [4]. Heaf and colleagues reported survival benefit for PD during the first two years of dialysis treatment from the Danish Terminal Uremia registry data [5]. Similar survival benefits were reported from Canadian cohort of dialysis patients initiating dialysis between 1990-1994 [6]. However, among CHOICE cohort, Jaar et al. reported no difference in the mortality risk during first year but significantly higher mortality risk for patients undergoing $\mathrm{PD}$ relative to HD during second year [7]. The CHOICE study has been criticized for collection of data after dialysis initiation, recruitment bias and data analysis. Mehrotra et al. examined the US Renal Data System (USRDS) for secular trends in survival among patients treated with HD and PD on day 90 of ESRD (HD 620, 020 patients; PD 64406 patients) in three 3-year cohorts (1996-1998, 1999-2001, and 2002-2004) for up to 5 years of follow up. Authors reported higher risk of death seen with PD in earlier cohorts which improved with time and there was no significant difference in the risk of death for PD and HD patients for the 2002-2004 cohorts during 5-year followup. Subgroups analysis based on age, diabetes status, and baseline comorbidity showed greater improvement in survival among patients treated with $\mathrm{PD}$ relative to $\mathrm{HD}$ at all follow-up periods [8]. In a separate study of 66,381 PD patients, comparing 1996-1998 cohorts to 2002-2004 cohorts, the risk of death and switch to HD were $45 \%$ and $38 \%$ lower, respectively, in the later cohort [9]. Researchers compared mortality rates between patients treated with PD and HD (including home HD) using data from 27,015 patients in the Australia and New Zealand Dialysis and Transplant Registry. Overall mortality rates were significantly lower during the 90 - to 365 -d period among those being treated with PD at day 90 (adjusted hazard ratio [HR] $0.89 ; P .001)$ [10]. Five-year survival on dialysis has improved significantly in last one decade. However, survival probabilities were improved more for patients on PD compared to HD. For incident patients on hemodialysis or peritoneal dialysis, survival probabilities in 1998-2002 were 7.2 and 14.8 percent higher, respectively, than in $1993-$ 1997 [1]. The improvement in survival for diabetics was 12.9 and 21.8 percent for HD and PD, respectively. Similarly for patients with hypertension, the improvement was 4.0 and 13.2 percent for $\mathrm{HD}$ and $\mathrm{PD}$, respectively [1]. In contrast to a common belief that survival on PD is inferior as compared to $\mathrm{HD}$, the converse is actually the case for most patient groups, particularly in the first few years of dialysis. Although the rates of native AVF are lower in PD patients, it has not translated into inferior survivals and it would be prudent to protect the veins of a PD patient specially those who are not a transplant candidate [11].

\section{Preservation of Residual Renal Function}

Residual renal function (RRF) is clinically important as it contributes to adequacy of dialysis, quality of life, and mortality in dialysis patients. Rate of decline in RRF is a more powerful prognostic factor than baseline RRF associated with all-cause mortality in PD patients [12]. Loss of RRF reduces small and middle molecular weight toxin clearance, decreased erythropoietin synthesis and increase sodium, phosphorus, and water retention [13]. These can lead to anemia, malnutrition, congestive heart failure, cardiac hypertrophy, atherosclerosis, vascular, and valvular calcification; and overall increase in cardiovascular morbidity and mortality [13]. In a study Moist et al. showed that PD had $65 \%$ lower risk of loss of RRF compared to HD during first year of RRT in incident ESRD patients [14]. Maintenance of RRF has shown direct correlation with patient survival. In a prospective observational study involving 231 chronic $\mathrm{PD}$ patients, Wang and colleagues reported $50 \%$ reduction in allcause mortality and cardiovascular death for every $1 \mathrm{ml} / \mathrm{min}$ increase in residual GFR [15]. It is imperative to periodically measure both the delivered dose of PD and RRF and make necessary adjustments to the dialysis prescription as RRF is lost. Efforts to maintain RRF with use of biocompatible PD solutions and treatment with ACE inhibitors should be considered [16].

\section{Lower Infection and Hospitalization Rates}

After cardiovascular disease, infection is the most common cause of death in dialysis patients accounting for 33 deaths per 1000 patient years in the USRDS cohort of 20012003 [1]. In a single-center observational cohort of dialysis patients ( $n=181$; HD 119 and PD 62) from 1999 to 2005, as expected only HD patients had bacteremia (0.16/year) and PD patients had peritonitis (0.24/year), the investigators however did not find any difference $(P=.24)$ in the rates of overall infection between $\mathrm{HD}$ and $\mathrm{PD}$ patients [17]. According to USRDS data from 2007, the rates of admission for bacteremia/septicemia were 1.5-2.3 times higher in HD patients reaching 102 per 1000 patient years compared to 66.7 per 1000 patient years in PD patients [1]. Comparing $\mathrm{HD}$ to PD as initial RRT modality, HD patients are at twice as higher risk of hospitalization from septicemia than PD patients. In another longitudinal cohort of incident dialysis patients with seven years of followup, the adjusted risk of death from septicemia was almost double in HD patients compared to PD patients (9.79 versus $4.81, P<.01$ ) [18]. Since the introduction of twin bags and Y set system and use of "flush before fill" technique, the rates of PD peritonitis have gone down significantly over the last few years. Use of Gentamicin cream application at exit site reduces the 
occurrence of both S Aureus and gram negative infections, including pseudomonas, and should be applied as a best practice [19].

\section{Quality of Life and Patient Satisfaction}

PD offers home-based therapy, thus there is no need to go to dialysis unit three times per week and no time spending in dialysis unit. Most PD patients pay visit to see their physician once a month. PD offers a flexible schedule with opportunities to travel and participate in other activities. Patient satisfaction is better amongst PD patients as compared those on HD [20]. In CHOICE cohort researchers surveyed 736 new dialysis patients from 37 centers regarding their satisfaction with treatment. Patients receiving PD were much more likely than those receiving $\mathrm{HD}$ to give excellent ratings of dialysis care overall and significantly more likely to give excellent ratings for each specific aspect of care rated. The differences between peritoneal dialysis and hemodialysis patient satisfaction persisted even after adjustment for patient age, race, education, health status, marital status, employment status, distance from the center, and time since starting dialysis [21].

\section{Better Graft Survival in Transplant Patient}

It would be preferable to start ESRD patients waiting for renal transplant on PD; especially ones with live donors. In a case-control study the incidence of delayed graft function was less, and the drop in serum creatinine was faster in continuous ambulatory peritoneal dialysis (CAPD) patients compared to HD patients. No differences in surgical complications or infections were observed between CAPD and HD patients [22]. One study reported high graft thrombosis with PD and high acute rejection episodes in HD patients but overall graft survival was similar [23]. While another study reported that the choice of pretransplant dialysis modality did not influence waiting time for transplant or the results of transplantation [24]. A study from the United States suggested that the use of PD immediately before transplantation predicts a 3\% lower risk for graft failure $(P<.05)$ and $6 \%$ lower risk for recipient death $(P<.001)$. Better recipient and graft survival was noted when patient received at least $50 \%$ of their pretransplant RRT time as $\mathrm{PD}$ [25].

\section{Economic Issues}

Per Center for Medicare and Medicaid, the cost difference between HD and PD was $\$ 13,900$ in 2001. Though the costs of both modalities have increased over years, the difference in cost has also increased to $\$ 20,000$ in 2009 [1]. Per person, per year cost for $\mathrm{HD}$ was $\$ 73,008$ versus $\$ 53,446$ for $\mathrm{PD}$ [1]. Until recently in the US, there was a fixed "composite rate" for dialysis-related services and in addition providers were also reimbursed for "injectable" medications (such as erythrocyte stimulating agents, intravenous iron, and vitamin D analogues) on an "as used" basis. On an average,
PD patient uses less intravenous medications than in-center HD patient which contributes to higher cost of HD.

Shih et al. evaluated the impact of initial dialysis modality choice and subsequent modality switches on Medicare expenditure in a 3-year period involving 3423 patients with new initiation of dialysis. After adjusting for patient characteristics, the annual Medicare expenditure was significantly lower for patients with peritoneal dialysis as the initial modality $(56,807$ dollars versus 68,253 dollars $)(P<.001)$. "Peritoneal dialysis, with at least one switch" and "hemodialysis, with at least one switch" had a lower or similar annual expenditure of $\$ 66,639$ and $\$ 72,335$, respectively [26]. In a recent cost comparison study of 463 dialysis patient with $12 \%$ of PD patients, the rate of hospitalization was less in PD patients compared to HD patients in the year following initiation of dialysis and had significantly lower health care cost over 12 month period. ( $\$ 173,507$ versus $\$ 129,997, P=$ .03) [27].

\section{Achieving Solute Clearance}

As with HD, the clearance of small solutes has been thought to be an important predictor of survival in PD patients. The Canada-USA (CANUSA) study examined the relationship between dialysis adequacy and mortality, hospitalization, and technique failure in PD patients. According to this study results, a decrease of $5 \mathrm{~L} / 1.75 \mathrm{~m}^{2}$ in creatinine clearance per week was associated with a $7 \%$ increase in relative risk of death. Also, a decrease of 0.1 units Kt/V per week was associated with a $5 \%$ increase in the relative risk of death. Based on these results, NKF-DOQI guidelines suggested a weekly $\mathrm{Kt} / \mathrm{V}$ of 2.0 and weekly creatinine clearance of $60 \mathrm{~L} / 1.73 \mathrm{~m}^{2}$ for PD patients. These Kt/V and creatinine clearance were difficult to achieve in large and anuric patients. However, the CANUSA study assumed that renal and peritoneal clearances were similar and additive [28]. The ADEMEX study showed that increasing peritoneal small solute clearance achievable in clinical practice did not improve survival in PD patients [29]. In a study from Hong Kong, Lo et al. showed that there was no difference in outcome for patients with $\mathrm{Kt} / \mathrm{Vurea}$ maintained above 2.0 versus Kt/Vurea between 1.7 and 2.0 [30]. Reanalysis of CANUSA data showed no association between peritoneal clearance and the relative risk of death. Based on these results, the 2006 NKF-DOQI guidelines suggested a minimum weekly Kt/Vurea target of 1.7 for PD. These lower adequacy targets are easier to achieve in practice and have not translated in inferior outcomes.

\section{Adequate Ultrafiltration and Volume Control}

Inability to achieve adequate ultrafiltration and failure to maintain volume homeostasis is another cause of technique failure in PD patients. Prevalence of ultrafiltration failure is reported to be between $1.7 \%$ and $13.7 \%$ [31]. Failure to follow fluid and dietary sodium restrictions necessitates use of more hypertonic solution to achieve adequate ultrafiltration. Use of hypertonic glucose solution can lead 
to hyperglycemia, weight gain, and hyperlipidemia. Furthermore, chronic exposure to hypertonic solutions which degrade into AGEs may alter peritoneal membrane transport characteristics, converting a low transporter into high transporter [32]. In two different PD cohorts-one from Netherlands and one from Japan-failure of ultrafiltration was the biggest reason for withdrawal from CAPD $[33,34]$. However, in these both cohorts the major modality was CAPD and Icodextrin was not used. Icodextrin is a glucose polymer that may be used as an osmotic alternative to dextrose in PD solutions. In a Japanese cohort of greater than 7000 patients, Kuriyama et al, showed that the dropout rate in patients who used Icodextrin $(8.9 \%)$, was significantly lower than those using dextrose (14.5\%), $(P<.0001)$ [35]. Newer biocompatible solutions without dextrose, and having a more physiologic $\mathrm{pH}$ have been associated with better preservation of peritoneal membrane; however, most human studies done with biocompatible solutions had short followup duration and clear evidence of benefit is lacking [36, 37]. Mechanical complications like catheter malposition or malfunction are also known to cause failure of ultrafiltration. Many causes of catheter malfunction such as occlusion by bladder or bowels can be corrected with use of laxatives or emptying the bladder. Obstruction due to clots can be dislodged with injecting heparinized saline; and if unsuccessful, by instillation of TPA or urokinase in the catheter. Common mechanical problems of omental trapping, adhesion formation, and so forth can be corrected through laparoscopic means by performing omentopexy, adhesiolysis, resection of epiploic appendices, colopexy, and so forth [38].

\section{Education Is Important}

Adequate understanding and education of patient and nephrologists are key to improve utilization rates of PD. In dialysis centers with limited knowledge and experience in performing PD, the recommendation "PD first" can lead to poor outcomes. Along with the education of physicians and medical staff, patients with chronic kidney disease approaching ESRD should be educated about kidney failure and renal replacement therapies. The National Pre-ESRD Education Initiative involved 15,000 patients from 932 referring nephrologists between 1997 and 2001. Upon completion of the program $55 \%$ chose hemodialysis, while $45 \%$ chose peritoneal dialysis, suggesting important influence of predialysis education on selection of modality of treatment [39].

Low prevalence of $\mathrm{PD}$ leads to inadequate exposure of in-training fellows to this modality. This in turn leads to vicious cycle in which uncomfortable nephrologists not offering PD to their patient and further reducing its use. To offset these concerns, the training programs must provide fellows adequate exposure to PD. Programs with limited access should offer fellows elective rotation in centers with larger PD population and have a core curriculum for PD including text and visual aids.

In conclusion, there is sufficient evidence to support the initiative of PD first and with improving PD techniques, greater use of biocompatible solutions, improved patient and physician education, and the bundling of dialysis services, providers will be more receptive towards the PD first initiative.

\section{References}

[1] "USRDS data system: USRDS annual data report," Bethesda MD, 2009.

[2] P. G. Blake and F. O. Finkelstein, "Why is the proportion of patients doing peritoneal dialysis declining in North America?" Peritoneal Dialysis International, vol. 21, no. 2, pp. 107-114, 2001.

[3] R. Mehrotra, D. Kermah, L. Fried et al., "Chronic peritoneal dialysis in the United States: declining utilization despite improving outcomes," Journal of the American Society of Nephrology, vol. 18, no. 10, pp. 2781-2788, 2007.

[4] E. F. Vonesh, J. J. Snyder, R. N. Foley, and A. J. Collins, "The differential impact of risk factors on mortality in hemodialysis and peritoneal dialysis," Kidney International, vol. 66, no. 6, pp. 2389-2401, 2004.

[5] J. G. Heaf, H. Løkkegaard, and M. Madsen, "Initial survival advantage of peritoneal dialysis relative to haemodialysis," Nephrology Dialysis Transplantation, vol. 17, no. 1, pp. 112$117,2002$.

[6] S. S. Fenton, D. E. Schaubel, M. Desmeules et al., "Hemodialysis versus peritoneal dialysis: a comparison of adjusted mortality rates," American Journal of Kidney Diseases, vol. 30, no. 3, pp. 334-342, 1997.

[7] B. G. Jaar, J. Coresh, L. C. Plantinga et al., "Comparing the risk for death with peritoneal dialysis and hemodialysis in a national cohort of patients with chronic kidney disease," Annals of Internal Medicine, vol. 143, no. 3, pp. 174-183, 2005.

[8] R. Mehrotra, Y.W. Chiu, K. Kalantar-Zadeh, J. Bargman, and E. Vonesh, "Similar outcomes with hemodialysis and peritoneal dialysis in patients with end-stage renal disease," Archives of Internal Medicine, vol. 171, no. 2, pp. 110-118, 2011.

[9] R. Mehrotra, Y. W. Chiu, K. Kalantar-Zadeh, and E. Vonesh, "The outcomes of continuous ambulatory and automated peritoneal dialysis are similar," Kidney International, vol. 76, no. 1, pp. 97-107, 2009.

[10] S. P. McDonald, M. R. Marshall, D. W. Johnson, and K. R. Polkinghorne, "Relationship between dialysis modality and mortality," Journal of the American Society of Nephrology, vol. 20, no. 1, pp. 155-163, 2009.

[11] D. J. Hirsch, K. K. Jindal, D. E. Schaubel, and S. S. Fenton, "Peritoneal dialysis reduces the use of non native fistula access in dialysis programs," Advances in Peritoneal Dialysis, vol. 15, pp. 121-124, 1999.

[12] C. T. Liao, Y. M. Chen, C. C. Shiao et al., "Rate of decline of residual renal function is associated with all-cause mortality and technique failure in patients on long-term peritoneal dialysis," Nephrology Dialysis Transplantation, vol. 24, no. 9, pp. 2909-2914, 2009.

[13] A. Y. Wang and K. N. Lai, "The importance of residual renal function in dialysis patients," Kidney International, vol. 69, no. 10, pp. 1726-1732, 2006.

[14] L. M. Moist, F. K. Port, S. M. Orzol et al., "Predictors of loss of residual renal function among new dialysis patients," Journal of the American Society of Nephrology, vol. 11, no. 3, pp. 556564, 2000.

[15] A. Y. Wang, M. Wang, J. Woo et al., "Inflammation, residual kidney function, and cardiac hypertrophy are interrelated and 
combine adversely to enhance mortality and cardiovascular death risk of peritoneal dialysis patients," Journal of the American Society of Nephrology, vol. 15, no. 8, pp. 2186-2194, 2004.

[16] P. K. Li, K. M. Chow, T. Y. Wong, C. B. Leung, and C. C. Szeto, "Effects of an angiotensin-converting enzyme inhibitor on residual renal function in patients receiving peritoneal dialysis: a randomized, controlled study," Annals of Internal Medicine, vol. 139, no. 2, pp. 105-112, 2003.

[17] N. Aslam, J. Bernardini, L. Fried, R. Burr, and B. Piraino, "Comparison of infectious complications between incident hemodialysis and peritoneal dialysis patients," Clinical Journal of the American Society of Nephrology, vol. 1, no. 6, pp. 12261233, 2006.

[18] N. R. Powe, B. Jaar, S. L. Furth, J. Hermann, and W. Briggs, "Septicemia in dialysis patients: incidence, risk factors, and prognosis," Kidney International, vol. 55, no. 3, pp. 1081-1090, 1999.

[19] F. H. Bender, J. Bernardini, and B. Piraino, "Prevention of infectious complications in peritoneal dialysis: best demonstrated practices," Kidney International Supplements, vol. 70, no. 103, pp. S44-S54, 2006.

[20] J. B. Wasserfallen, M. Moinat, G. Halabi et al., "Satisfaction of patients on chronic haemodialysis and peritoneal dialysis," Swiss Medical Weekly, vol. 136, no. 13-14, pp. 210-217, 2006.

[21] H. R. Rubin, N. E. Fink, L. C. Plantinga, J. H. Sadler, A. S. Kliger, and N. R. Powe, "Patient ratings of dialysis care with peritoneal dialysis vs hemodialysis," Journal of the American Medical Association, vol. 291, no. 6, pp. 697-703, 2004.

[22] R. Vanholder, P. Heering, A. Van Loo et al., "Reduced incidence of acute renal graft failure in patients treated with peritoneal dialysis compared with hemodialysis," American Journal of Kidney Diseases, vol. 33, no. 5, pp. 934-940, 1999.

[23] R. Palomar, P. Morales, E. Rodrigo et al., "Venous graft thrombosis in patients on peritoneal dialysis before transplantation," Transplantation Proceedings, vol. 39, no. 7, pp. 2128-2130, 2007.

[24] Y. Chalem, J. P. Ryckelynck, P. Tuppin, C. Verger, S. Chauvé, and D. Glotz, "Access to, and outcome of, renal transplantation according to treatment modality of end-stage renal disease in France," Kidney International, vol. 67, no. 6, pp. 2448-2453, 2005.

[25] A. S. Goldfarb-Rumyantzev, J. F. Hurdle, J. D. Scandling, B. C. Baird, and A. K. Cheung, "The role of pretransplantation renal replacement therapy modality in kidney allograft and recipient survival," American Journal of Kidney Diseases, vol. 46, no. 3, pp. 537-549, 2005.

[26] Y. C. Shih, A. Guo, P. M. Just, and S. Mujais, "Impact of initial dialysis modality and modality switches on Medicare expenditures of end-stage renal disease patients," Kidney International, vol. 68, no. 1, pp. 319-329, 2005.

[27] A. Berger, J. Edelsberg, G. W. Inglese, S. K. Bhattacharyya, and G. Oster, "Cost comparison of peritoneal dialysis versus hemodialysis in end-stage renal disease," American Journal of Managed Care, vol. 15, no. 8, pp. 509-518, 2009.

[28] "Adequacy of dialysis and nutrition in continuous peritoneal dialysis: association with clinical outcomes. Canada-USA (CANUSA) Peritoneal Dialysis Study Group," Journal of the American Society of Nephrology, vol. 7, no. 2, pp. 198-207, 1996.

[29] R. Paniagua, D. Amato, E. Vonesh et al., "Effects of increased peritoneal clearances on mortality rates in peritoneal dialysis:
ADEMEX, a prospective, randomized, controlled trial," Journal of the American Society of Nephrology, vol. 13, no. 5, pp. 1307-1320, 2002.

[30] W. K. Lo, Y. W. Ho, C. S. Li et al., "Effect of Kt/V on survival and clinical outcome in CAPD patients in a randomized prospective study," Kidney International, vol. 64, no. 2, pp. 649-656, 2003.

[31] P. J. Margetts and D. N. Churchill, "Acquired ultrafiltration dysfunction in peritoneal dialysis patients," Journal of the American Society of Nephrology, vol. 13, no. 11, pp. 2787-2794, 2002.

[32] M. L. Lambie, B. John, L. Mushahar, C. Huckvale, and S. J. Davies, "The peritoneal osmotic conductance is low well before the diagnosis of encapsulating peritoneal sclerosis is made," Kidney International, vol. 78, no. 6, pp. 611-618, 2010.

[33] K. J. Jager, M. P. Merkus, F. W. Dekker et al., "Mortality and technique failure in patients starting chronic peritoneal dialysis: results of the netherlands cooperative study on the adequacy of dialysis," Kidney International, vol. 55, no. 4, pp. 1476-1485, 1999.

[34] Y. Kawaguchi, T. Hasegawa, M. Nakayama, H. Kubo, and T. Shigematu, "Issues affecting the longevity of the continuous peritoneal dialysis therapy," Kidney International, vol. 51, no. 62, pp. S105-S107, 1997.

[35] R. Kuriyama, A. Tranaeus, and T. Ikegami, "Icodextrin reduces mortality and the drop-out rate in japanese peritoneal dialysis patients," Advances in Peritoneal Dialysis, vol. 22, pp. 108-110, 2006.

[36] H. Y. Choi, D. K. Kim, T. H. Lee et al., “The clinical usefulness of peritoneal dialysis fluids with neural $\mathrm{pH}$ and low glucose degradation product concentration: an open randomized prospective trial," Peritoneal Dialysis International, vol. 28, no. 2, pp. 174-182, 2008.

[37] B. Rippe, O. Simonsen, O. Heimbürger et al., "Long-term clinical effects of a peritoneal dialysis fluid with less glucose degradation products," Kidney International, vol. 59, no. 1, pp. 348-357, 2001.

[38] J. H. Crabtree and R. J. Burchette, "Effective use of laparoscopy for long-term peritoneal dialysis access," American Journal of Surgery, vol. 198, no. 1, pp. 135-141, 2009.

[39] T. Golper, "Patient education: can it maximize the success of therapy?" Nephrology Dialysis Transplantation, vol. 16, supplement 7, pp. 20-24, 2001. 


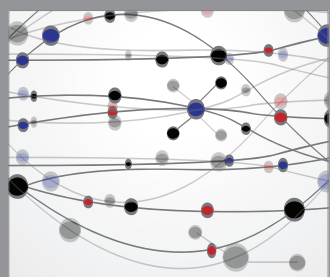

The Scientific World Journal
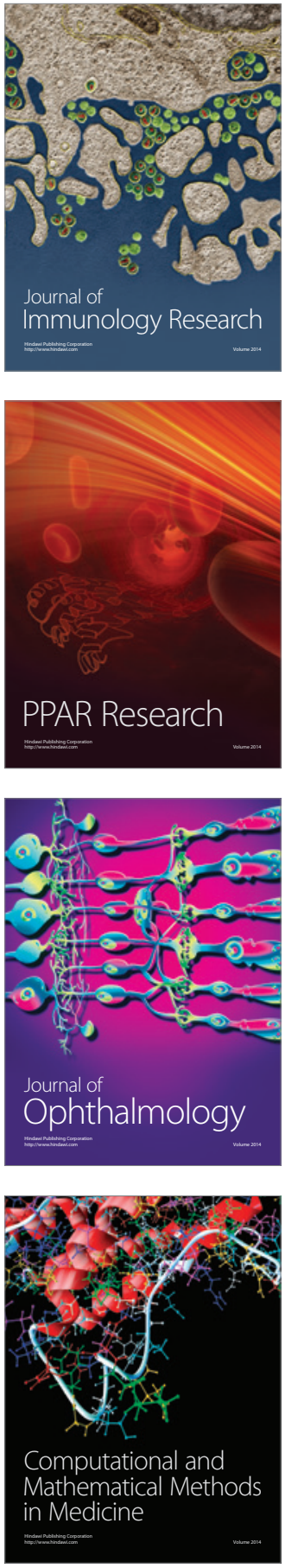

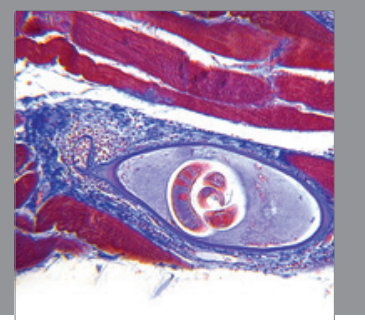

Gastroenterology

Research and Practice
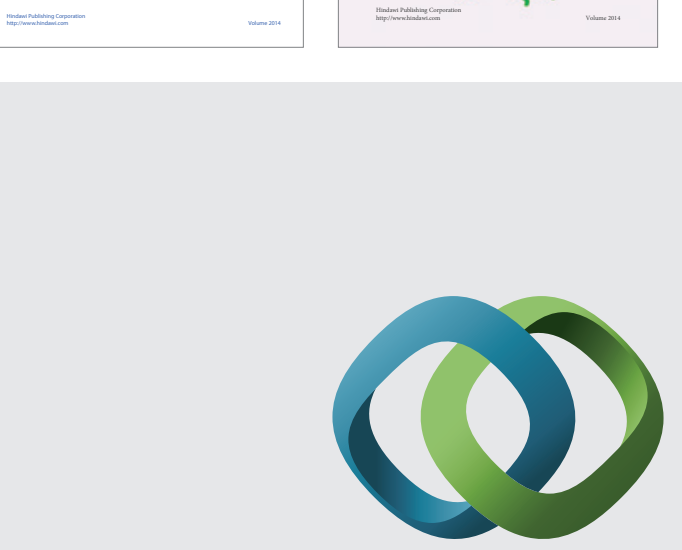

\section{Hindawi}

Submit your manuscripts at

http://www.hindawi.com
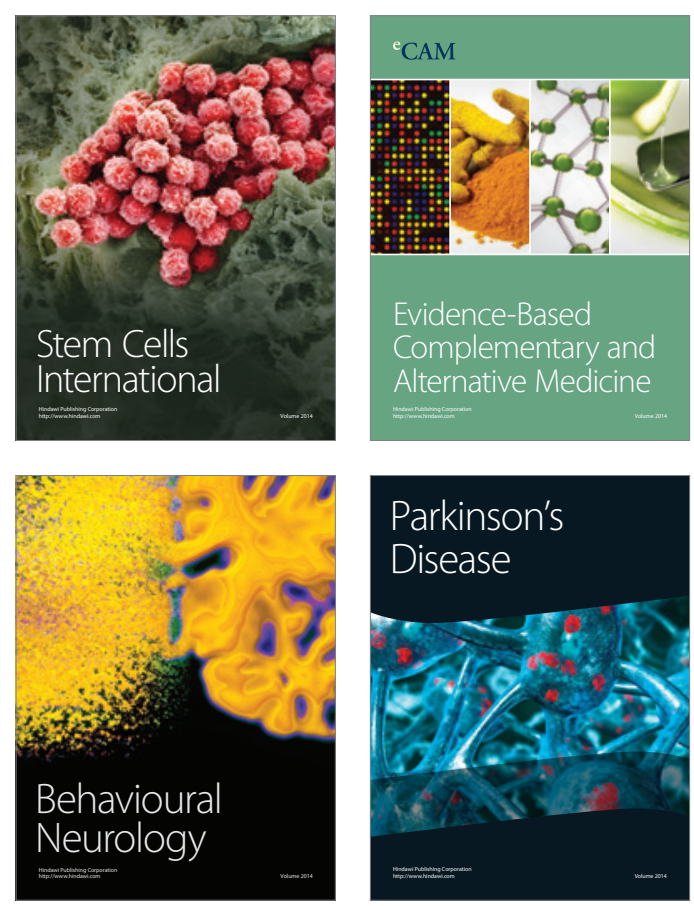

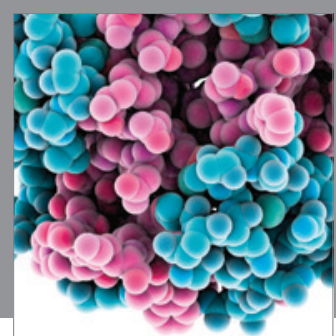

Journal of
Diabetes Research

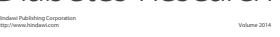

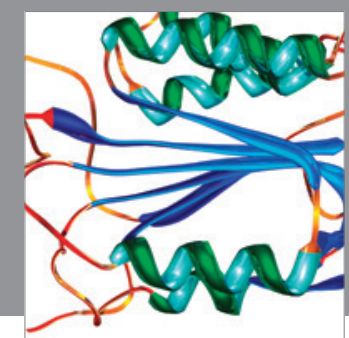

Disease Markers
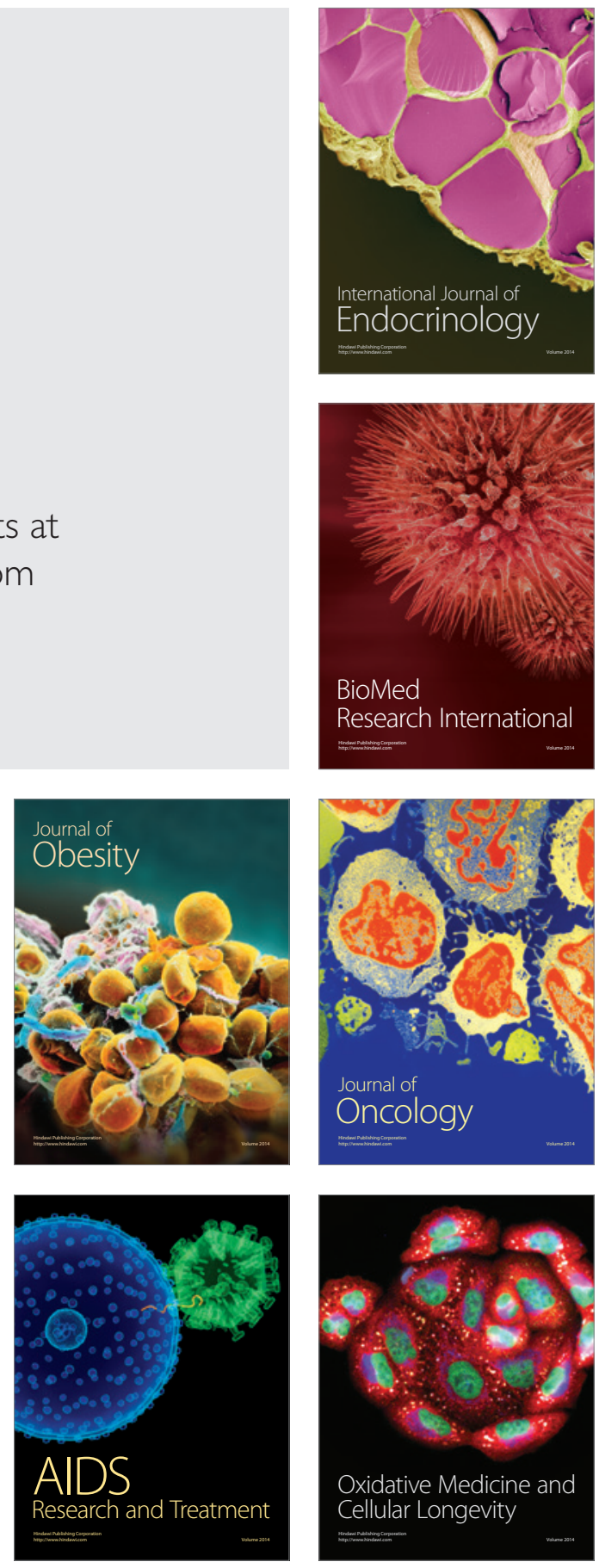